\title{
Mapping the Potential of Zero Charge (PZC) and Electrocatalytic Activity of Metal- Electrolyte Interface via a Grain-by-Grain Approach
}

Yufei Wang, Emma Gordon, and Hang Ren*

Department of Chemistry and Biochemistry, Miami University, Oxford, $\mathrm{OH} 45056$, United States

* renhang@miamioh.edu

\section{Supporting Information}

\section{Table of Contents}

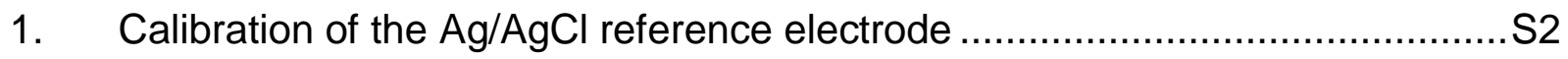

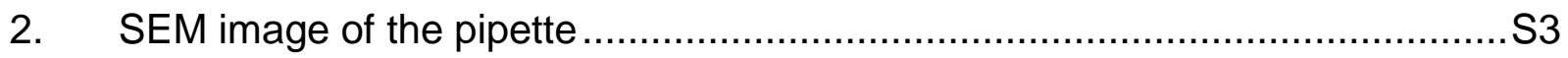

3. Stray capacitance and instrument response ……….................................. 4

4. Charge from double layer charging vs monolayer adsorption ........................S6

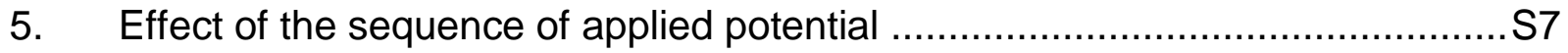

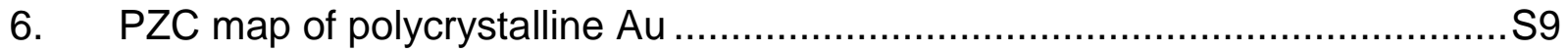

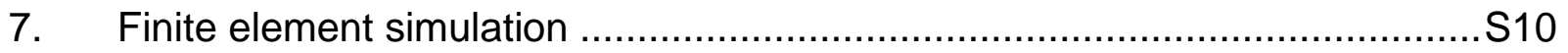

8. Additional correlated PZC and electroactivity maps on polycrystalline Pt ......S19

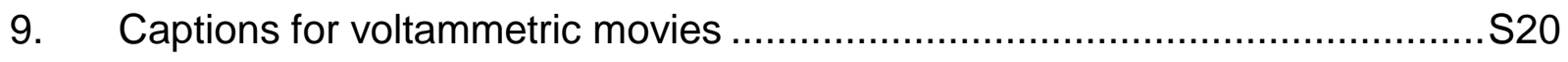

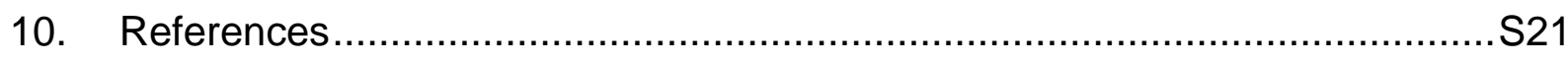




\section{Calibration of the $\mathrm{Ag} / \mathrm{AgCl}$ reference electrode}

As described in the experimental section, the potential of the reference electrode was calibrated after each experiment to RHE. This is done by measuring the open circuit potential between the leakless $\mathrm{Ag} / \mathrm{AgCl}$ and a Pt microelectrode immersed in the solution of the same composition used in SECCM under $1 \mathrm{~atm} \mathrm{H}_{2}$. An example showing the open circuit potential during the calibration of $\mathrm{Ag} / \mathrm{AgCl}$ in $1 \mathrm{mM} \mathrm{HClO} 4$ to $\mathrm{RHE}$ is show in Figure S1, which yield $0.410 \mathrm{~V}$ for $E_{\mathrm{Ag} / \mathrm{AgCl}} \mathrm{Vs} \mathrm{RHE}$.

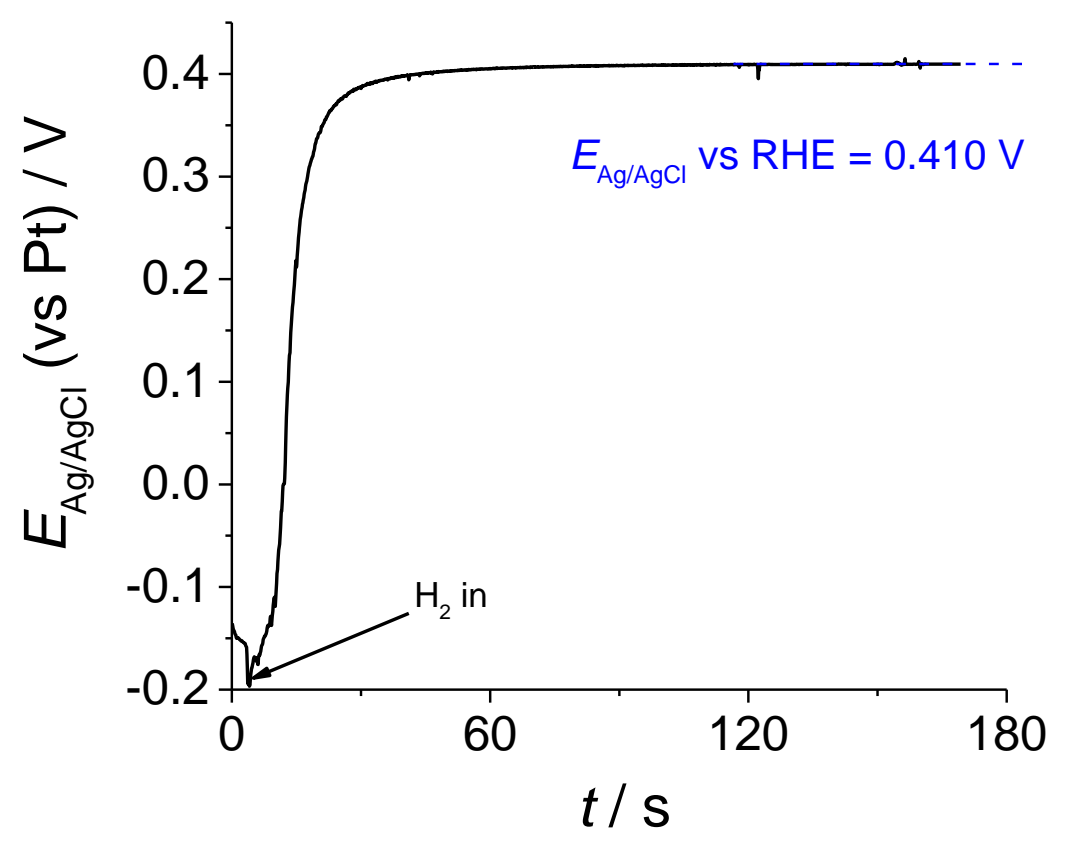

Figure S1. Calibration of the leakless $\mathrm{Ag} / \mathrm{AgCl}$ reference electrode to an $\mathrm{RHE}$ electrode by measuring the open circuit potential between the $\mathrm{Ag} / \mathrm{AgCl}$ and $\mathrm{Pt}$ in $1 \mathrm{mM} \mathrm{HClO} 4$ under $1 \mathrm{~atm} \mathrm{H}_{2}$. 


\section{SEM image of the pipette}

The opening of the tip of the pipette, as well as the half cone angle of the pipette was measured by FE-SEM. An example is shown in Figure S2. The opening of the pipette is $\sim 940 \mathrm{~nm}$ in diameter. The half-cone angle measured from the SEM image is $9^{\circ}$.
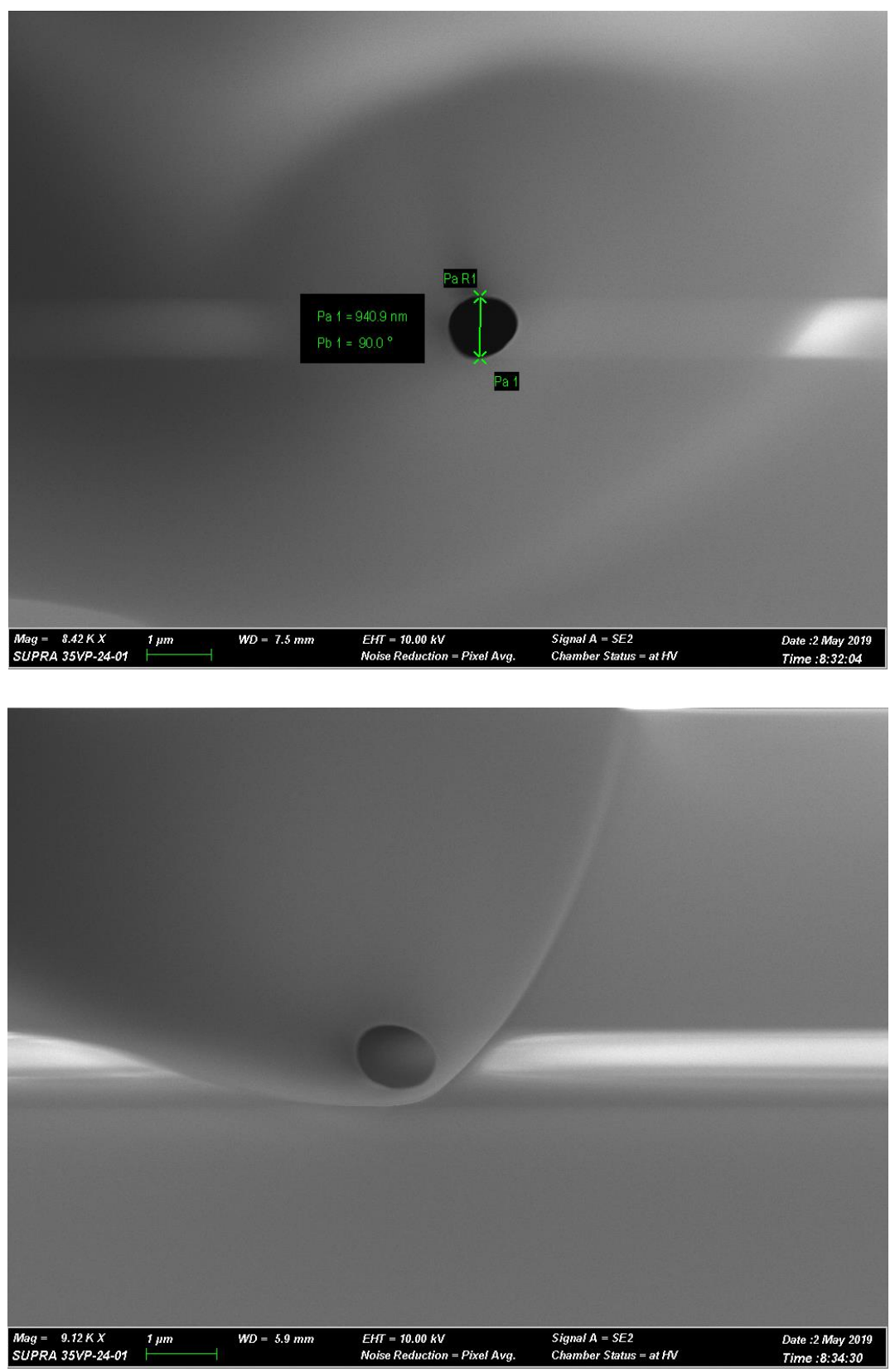

Figure S2. SEM images showing the opening of a typical pipette used in the SECCM experiment. 


\section{Stray capacitance and instrument response}

The instrument response is measured using a model circuit (HEKA MC10), which is composed of a $5.1 \mathrm{M} \Omega$ resistor in series with a $22 \mathrm{pF}$ capacitor that is in parallel with 0.5 $\mathrm{G} \Omega$ resistor. As shown in Figure S3, the measured exponential decay constant (114 $\mu \mathrm{s})$ from the current transient during a potential step from $0 \mathrm{~V}$ to $0.1 \mathrm{~V}$ is highly consistent (within $2 \%$ of relative deviation) with the time constant of the circuit $(112 \mu \mathrm{s}=5.1 \mathrm{M} \Omega \times$ $22 \mathrm{pF}$ ). The consistency between the expected and measured time constant in the RC circuit indicates that the instrument response is faster than $112 \mu \mathrm{s}$. This also suggests that effect of stray capacitance in measuring a $22 \mathrm{pF}$ capacitor is minimal. Even assuming a $5 \%$ estimated tolerance in the value of $\mathrm{RC}$, the stray capacitance in parallel with the model circuit is smaller than $1.1 \mathrm{pF}$. This value is smaller than the double layer capacitance calculated in section 4 of the SI.

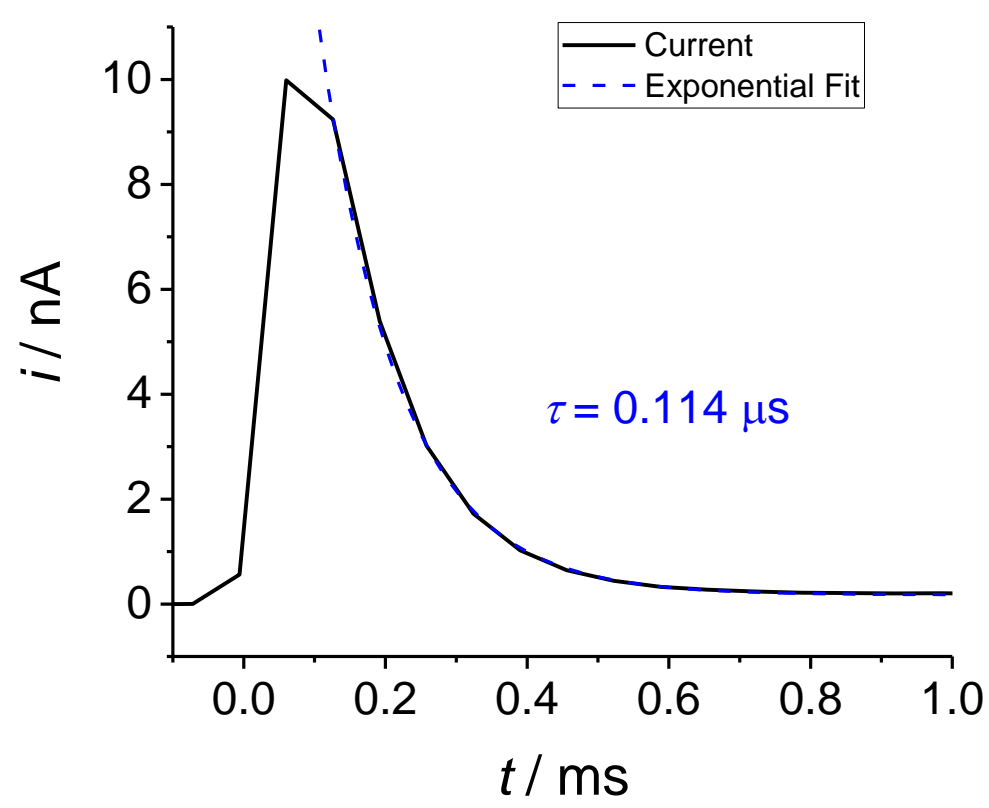

Figure S3. The amplifier response from a $0.1 \mathrm{~V}$ potential step on a model $\mathrm{RC}$ circuit. The blue dash line is an exponential fit with a decay constant of $0.114 \mu \mathrm{s}$. 
We also explored the effect of instrument filter on the shape and charge associated with the current spikes. To this end, the same pipette is approached to a Pt held at $0.1 \mathrm{~V}$ vs $\mathrm{Ag} / \mathrm{AgCl}$ with current recorded at different sampling/filter frequencies. Overall, the spike returns close to the baseline level within the first $5 \mathrm{~ms}$, and the shape of the spike is only slightly affected (Figure S4). The charge within the first $5 \mathrm{~ms}$ is between 0.1 to 0.2 $\mathrm{pC}$, which is comparable with the charge associated the non-Faradaic charging current. This suggest that the charge can be measured within the first $5 \mathrm{~ms}$ even if a $10 \mathrm{kHz}$ filter is used. To store data more efficiently and reduce the noise in the scanning hopping experiment for PZC measurement, we use a $10 \mathrm{kHz}$ 6-pole Bessel filter for the scanning experiments, and use the first $5 \mathrm{~ms}$ of the spike to estimate the charge associated with the formation of the electrical double layer at the electrode-electrolyte interface.
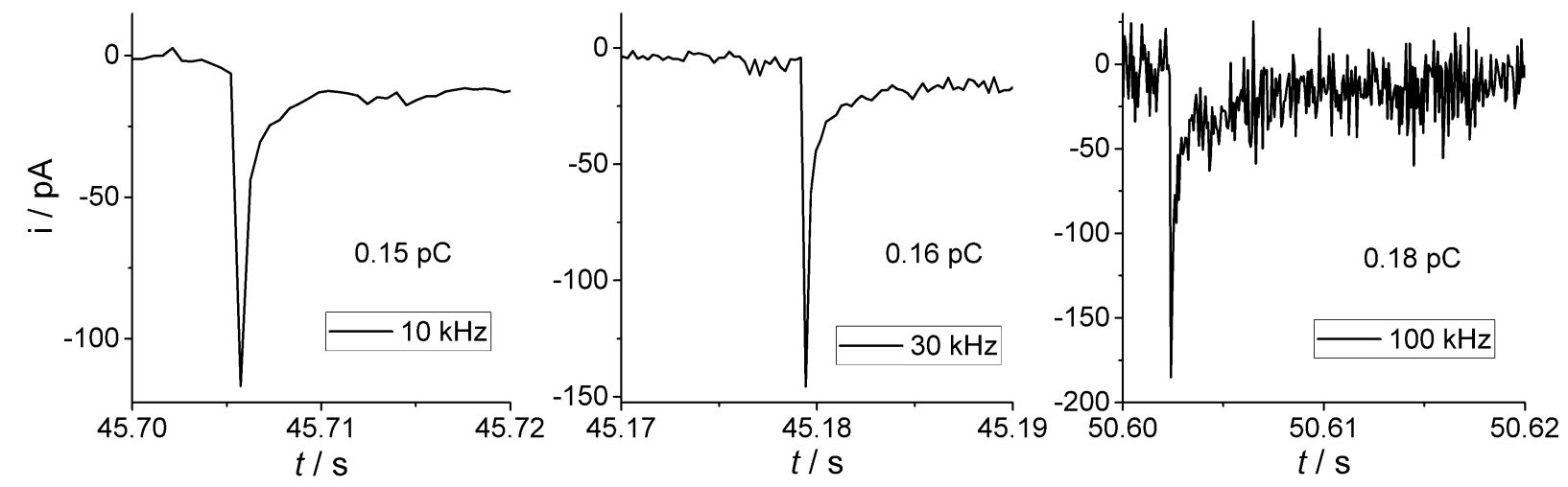

Figure S4. Effect of filter frequency on the current spike when approaching a pipette to Pt electrode held at $0.35 \mathrm{~V}$ vs SHE. The filters are analog 6-pole Bessel low pass filters. The charge within the first $5 \mathrm{~ms}$ of the spike are specified in the figure. 


\section{Charge from double layer charging vs monolayer adsorption}

The capacitance of the droplet-metal interface can be estimated using the double layer capacitance of $\mathrm{Pt}\left(C_{d}=20 \mu \mathrm{F} \mathrm{cm}^{-2}\right) .{ }^{1}$ Using the surface area of the droplet footprint of $3.2 \times 10^{-8} \mathrm{~cm}^{2}$ (based on the SEM image shown in Figure 2), the double layer capacitance is estimated to be $1.4 \mathrm{pF}$. The charge for charging the capacitor by a $0.1 \mathrm{~V}$ voltage step is $1.4 \times 10^{-13} \mathrm{C}$ according to $Q=C V$.

On the other hand, the charge within the first $5 \mathrm{~ms}$ of the current spike can have contribution from Faradaic process. Herein, we also estimate the charge associated with the adsorption of a monolayer species. Using $200 \mu \mathrm{C} \mathrm{cm}^{-2}$ for monolayer adsorption and without considering the roughness of the surface, the charge expected for forming a monolayer at the electrode surface in an SECCM experiment is at least $1.5 \times 10^{-11} \mathrm{C}$. Note that this charge is two orders of magnitude smaller than the charge integrated in our experiment. Therefore, the charge associated with the spike is likely mostly contributed by the charging of the electrical double layer. 


\section{Effect of the sequence of applied potential}

In the main text, we mentioned that the electrode potential after the approach needs to be scanned back to the double layer region to obtain consistent PZC values. To demonstrate, we studied the effect the direction of potential applied had on the value of PZC determined. A pipette filled with $\mathrm{HClO}_{4}$ was approached Pt surface using two different voltage sequences: an ascending voltage sequence and a descending voltage sequence. The voltage profiles are shown in panels B and C of Figure S5. After each approach, the electrode potential remained unchanged before the next approach. From repetitive approach at 15 different locations on $\mathrm{Pt}$, the average $\mathrm{PZC}$ determined is 0.71 $\mathrm{V}$ and $0.66 \mathrm{~V}$ vs SHE for ascending and descending voltages, respectively. We hypothesized this $50 \mathrm{mV}$ difference is attributed to the hysteresis in the adsorption of $\mathrm{H}$ and $\mathrm{OH}$ on $\mathrm{Pt}$.

In contrast, if the electrode potential after each approach is swept back to a fixed value that lies in the range of double layer region of $\mathrm{Pt}\left(V_{0}\right)$, the $\mathrm{PZC}$ determined using either an ascending voltage sequence (Figure S5D) or a descending voltage sequence show very similar measured PZC values from measurement of 15 positions. This is likely due to the renewal of the electrode surface via the removal the adsorbed $\mathrm{H}$ or $\mathrm{OH}$ when the potential is stepped back to the double layer region before the next approach occurred. 

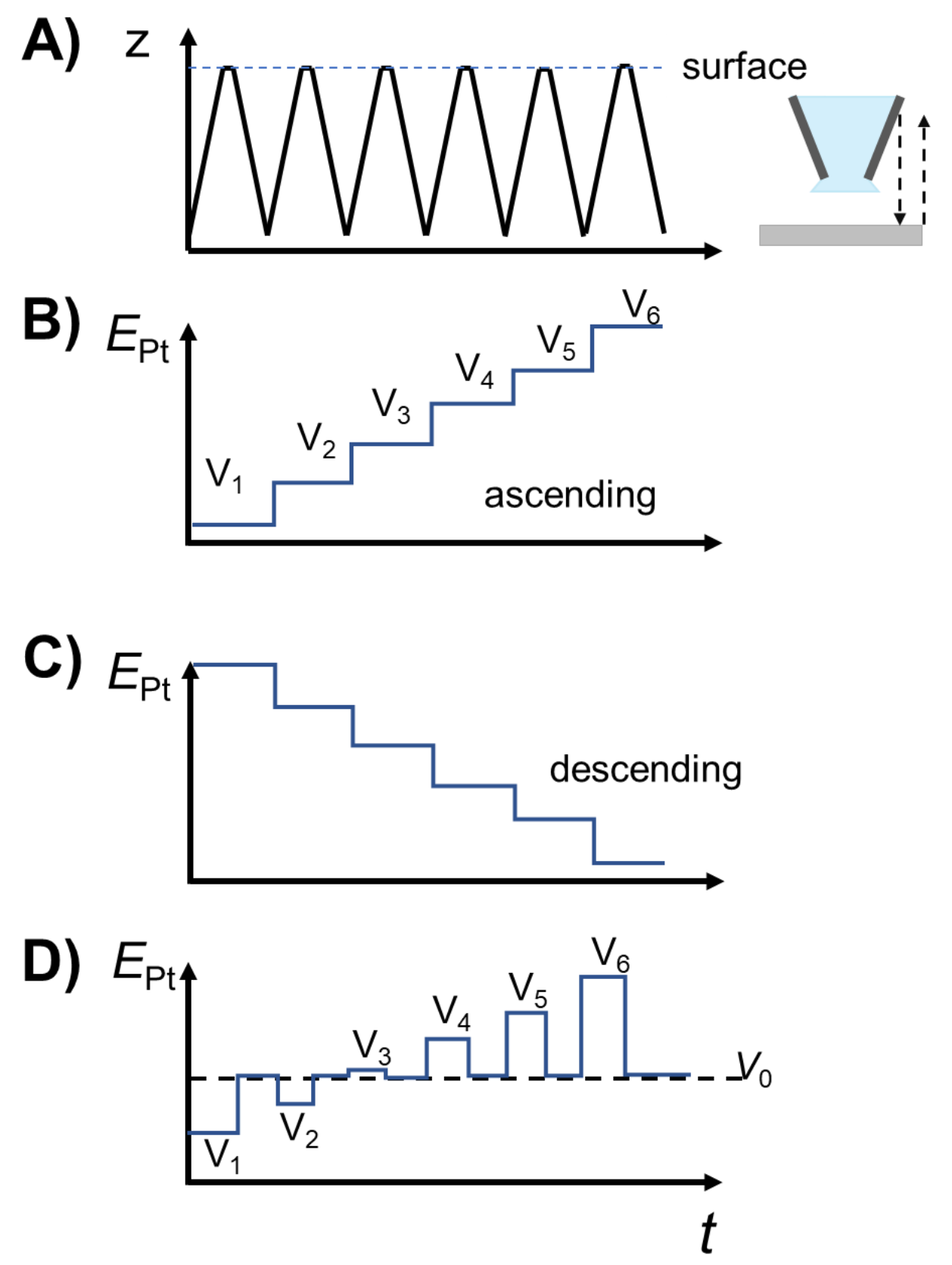

Figure S5. A) pipette vertical position ( $z$ ) vs time showing the repetitive approaching of the pipette to the same location of a substrate. Voltage profile applied to Pt during repetitive approaching at a single location in $B$ ) ascending voltage sequence and $C$ ) descending voltage sequence. D) substrate potential is swept back to a fixed potential in the double layer region of $\mathrm{Pt}\left(\mathrm{V}_{0}\right)$ during each approach/retraction. 


\section{PZC map of polycrystalline Au}

To further validate our method, we also mapped the PZC on a polycrystalline Au by repetitive approaching SECCM. An SEM image showing the footprint left by the SECCM scan is shown in Figure S6a. At least two apparent grains are observed in the SEM image (Figure S6a, color added for clarity). Similarly, in the PZC map (Figure S6b), the two regions show different $P Z C$ values. The distribution of $P Z C$ values can also be seen from the histogram in Figure S6c, which displays a bimodal distribution with peak centers at $\sim 0.17 \mathrm{~V}$ and $\sim 0.22 \mathrm{~V}$ vs SHE respectively. This measured PZC on Au agrees well with those obtained on single crystal Au surface using capacitance measurement. ${ }^{2}$
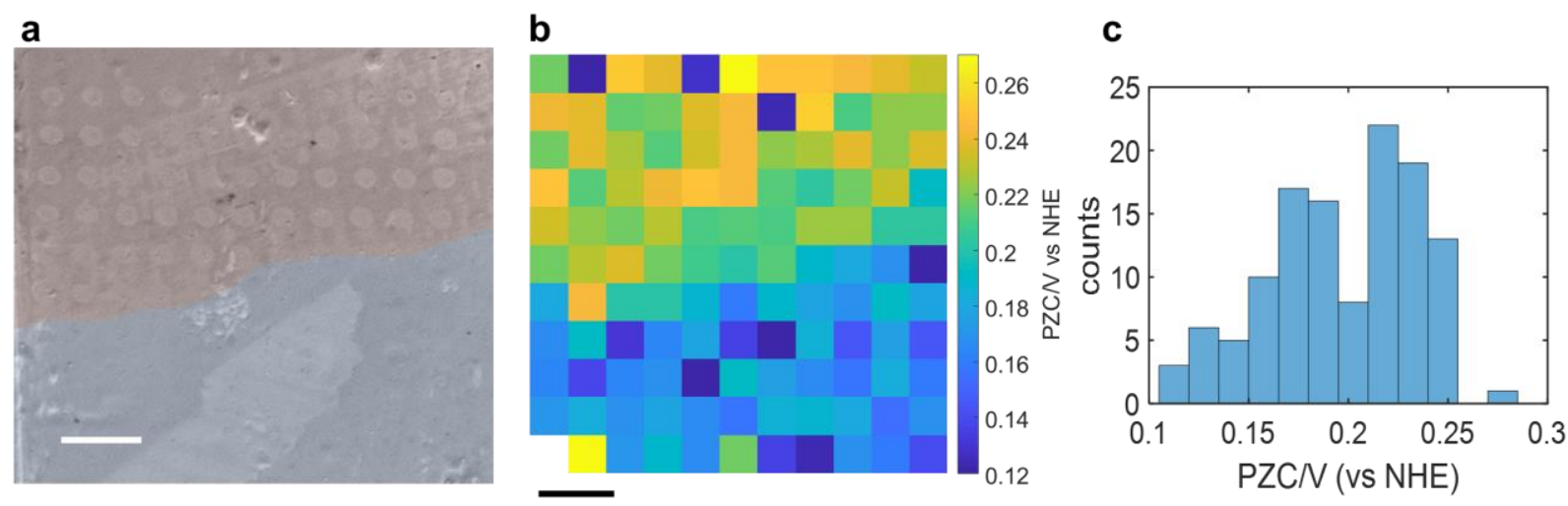

Figure S6. a) SEM image showing the area scanned in the SECCM. Note that footprint is only clear on the grain at the top of the image. Color is added for clarity. b) PZC map on polycrystalline Au measured by SECCM using repetitive approaching. c) histogram showing the distribution PZC obtained on Au. The scale bar is $10 \mu \mathrm{m}$. 


\section{Finite element simulation}

We used finite element simulation to extract the kinetics of electron transfer during HER. The axial symmetry of the single barrel pipette was considered in the simulation. Timedependent simulation was performed to obtain the voltammogram. The concentrations of $\mathrm{H}^{+}$and $\mathrm{H}_{2}$ were determined in the 2-D axial geometry shown in Figure $\mathrm{S} 6$ by Fick's second law:

$$
\begin{aligned}
\frac{\partial C_{\mathrm{H}^{+}}}{\partial t} & =D_{\mathrm{H}^{+}} \nabla^{2} C_{\mathrm{H}^{+}} \\
\frac{\partial C_{\mathrm{H}_{2}}}{\partial t} & =D_{\mathrm{H}_{2}} \nabla^{2} C_{\mathrm{H}_{2}}
\end{aligned}
$$

The boundary conditions are specified in Figure S7. Butler Volmer kinetics is invoked to determine the flux at the electrode surface as a function of potential. Because of the small half cone angle (measured from SEM), a sufficiently long portion of the pipette is simulated to ensure the accuracy of the time-dependent simulation. The height of the droplet a wide range (from $20 \%$ of the radius to $160 \%$ of the radius) has found to have negligible effect on the limiting current (data not shown) and is taken as $50 \%$ of the radius of the contact in the simulation.

Faradaic current from the simulation is obtained by integrating the normal flux of $\mathrm{H}^{+}$ $\left(\mathrm{H}^{+}\right)$across the electrode surface multiplied by Faraday's constant:

$$
i=F \iint J_{\mathrm{H}^{+}} \mathrm{d} A
$$

The effect of $k^{0}$ on the shape of the voltammogram is shown in Figure S8. Note that only the potential scan in the negative direction is shown. The voltammogram reaches quasi-steady state at $1 \mathrm{~V} / \mathrm{s}$, consistent with the experiments.

To fit data and extract the parameters in Butler-Volmer, we evaluate five potentials on the voltammogram: $E_{0.10}, E_{0.25}, E_{0.50}, E_{0.75}, E_{0.90}$, corresponding to potentials at $10 \%$, $25 \%, 50 \%, 75 \%$ and $90 \%$ of the limiting current, respectively. The effect of transfer coefficient $(\alpha)$ and heterogeneous electron transfer rate constant $\left(k^{0}\right)$ on these five potentials are summarized in 2D color plots in Figures S9 -S13. 
To extract the kinetic parameters ( $\alpha$ and $k^{0}$ ) of HER observed in SECCM experiments,

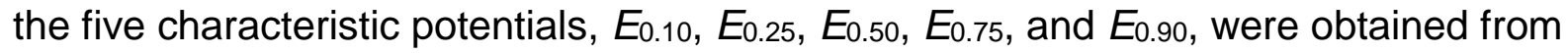
the experimental voltammogram. An optimization was performed in MATLAB 2018b to search $\alpha$ and $k^{0}$ that minimize the mean square error of the five characteristic potentials between the experiment and the simulation. Examples of the experimental and best fit voltammograms are shown in Figure S14. A heatmap showing the optimized $\alpha$ and $k^{0}$ from each position scanned are shown in Figure S15.
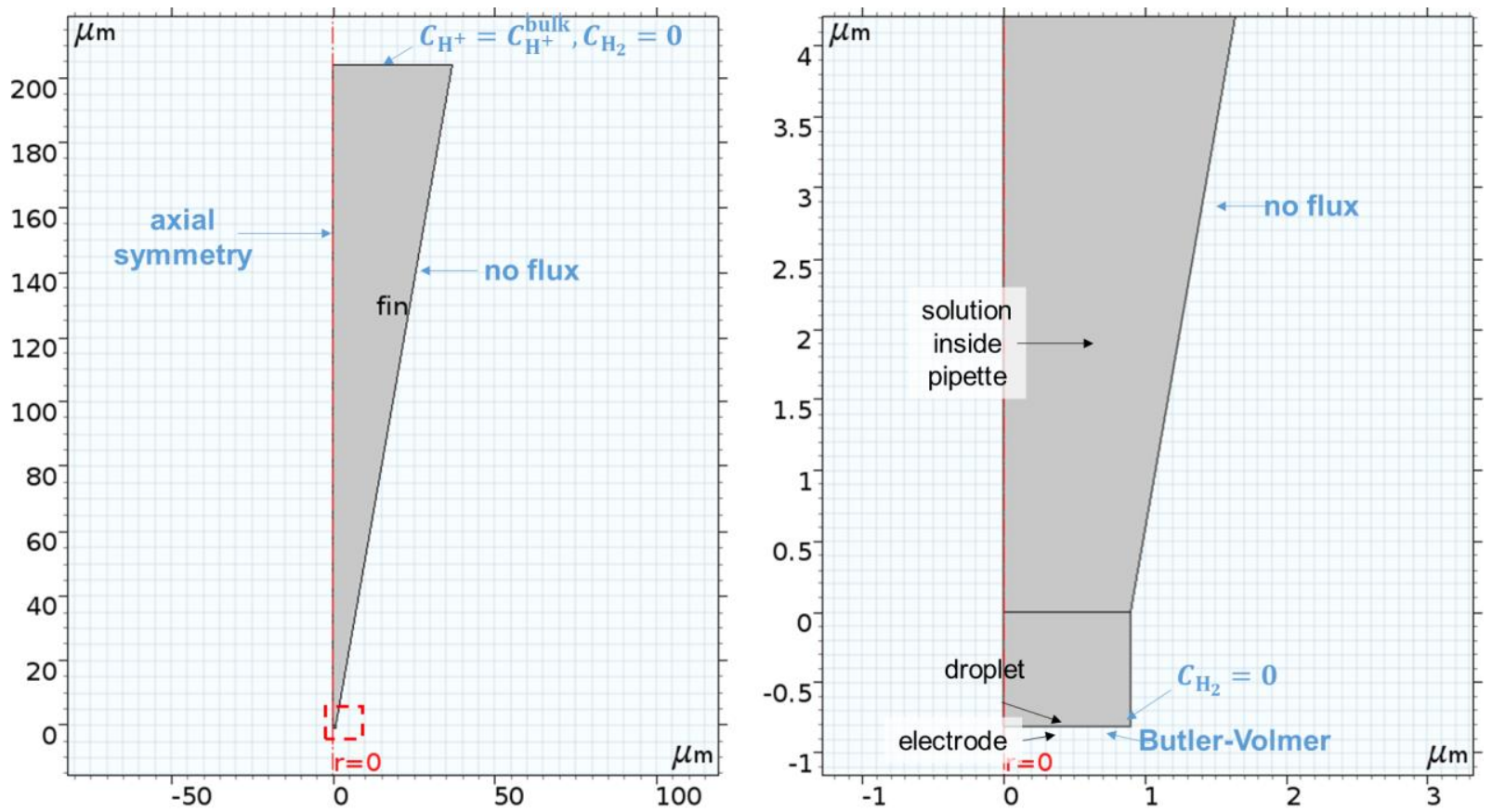

Figure S7. The geometry and boundary conditions used for finite element analysis. The boundary conditions were also labeled (blue). 


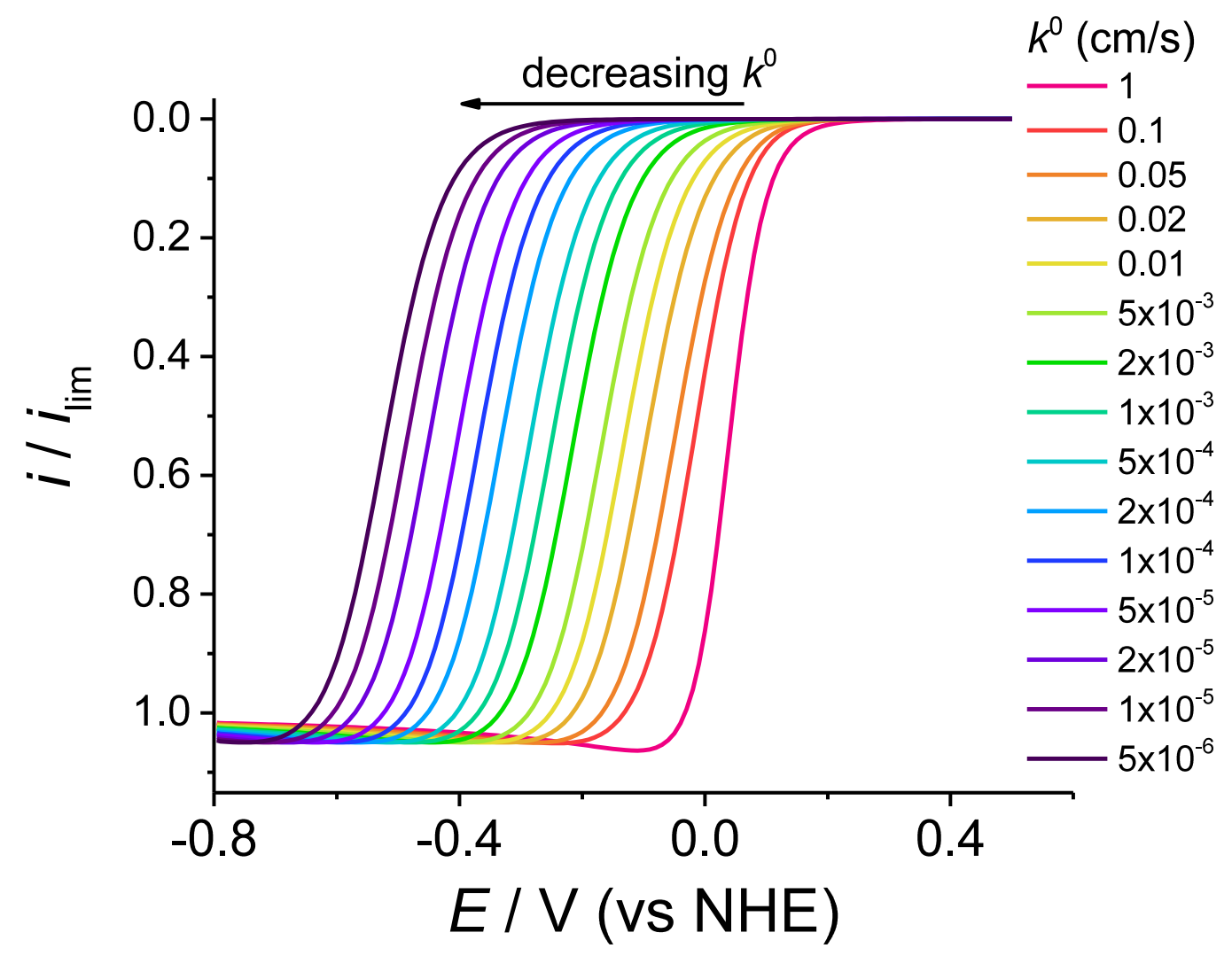

Figure S8. Simulated voltammograms for hydrogen evolution reaction in $10 \mathrm{mM} \mathrm{HClO}_{4}$ with different $k^{0}$. Scan rate is $1 \mathrm{~V} / \mathrm{s}$. Only the first segment, sweeping from $0.5 \mathrm{~V}$ to -0.8 $\mathrm{V}$, is shown. 


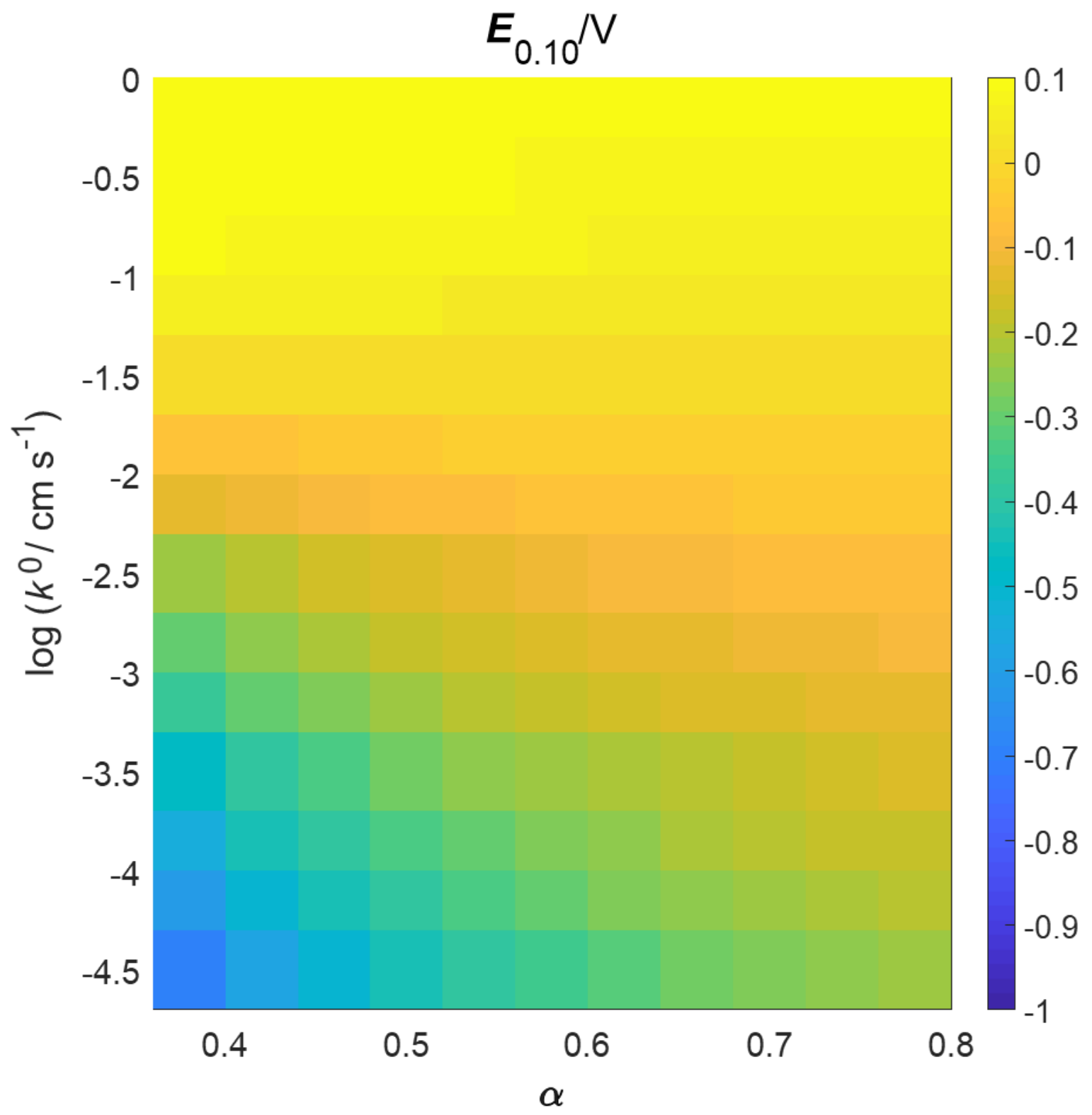

Figure S9. Effect of $\alpha$ and $k^{0}$ on the potential corresponding to $10 \%$ of the limiting current $\left(E_{0.10}\right)$ from the simulated SECCM voltammogram. 


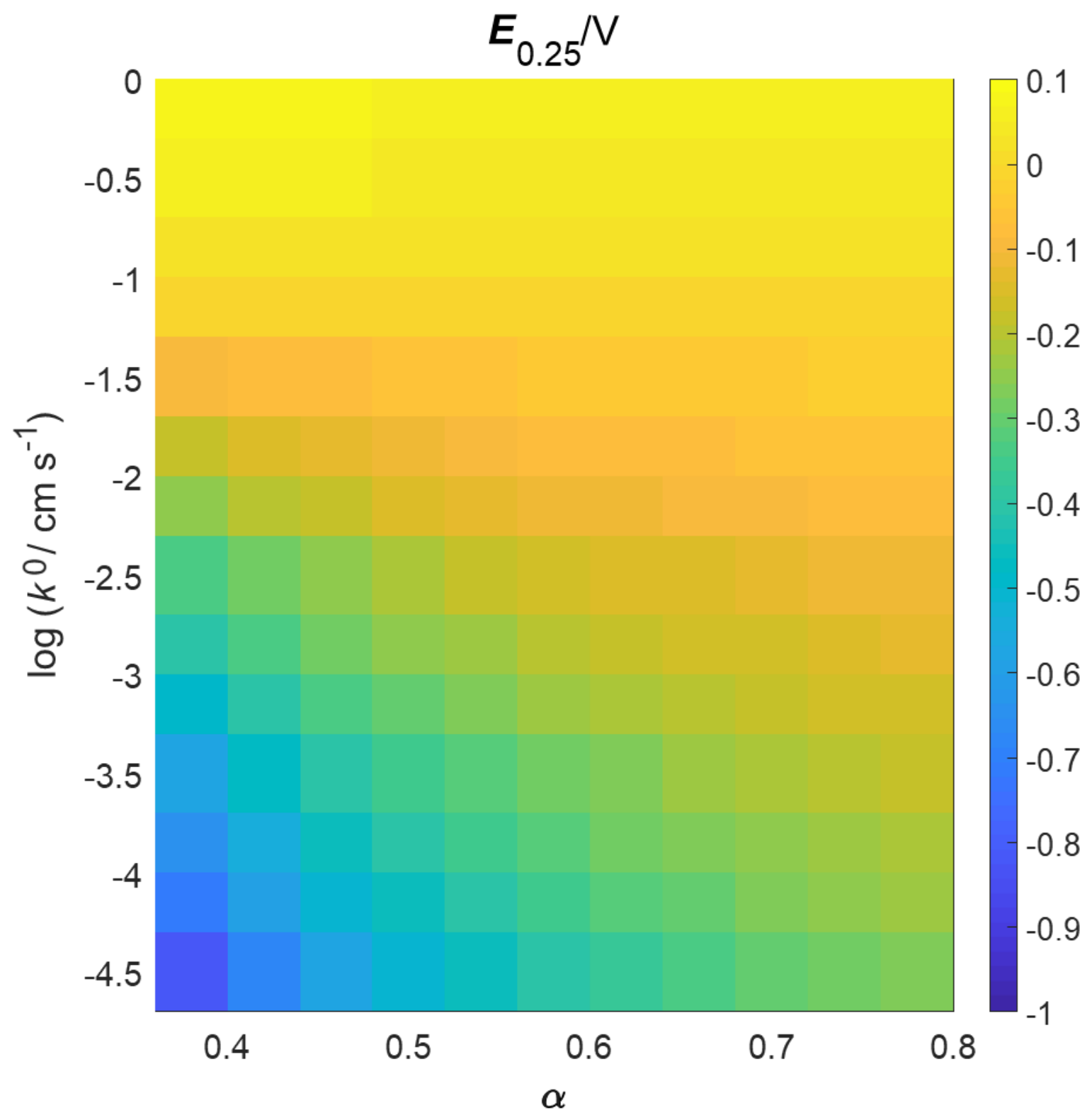

Figure S10. Effect of $\alpha$ and $k^{0}$ on the potential corresponding to $25 \%$ of the limiting current $\left(E_{0.25}\right)$ from the simulated SECCM voltammogram. 


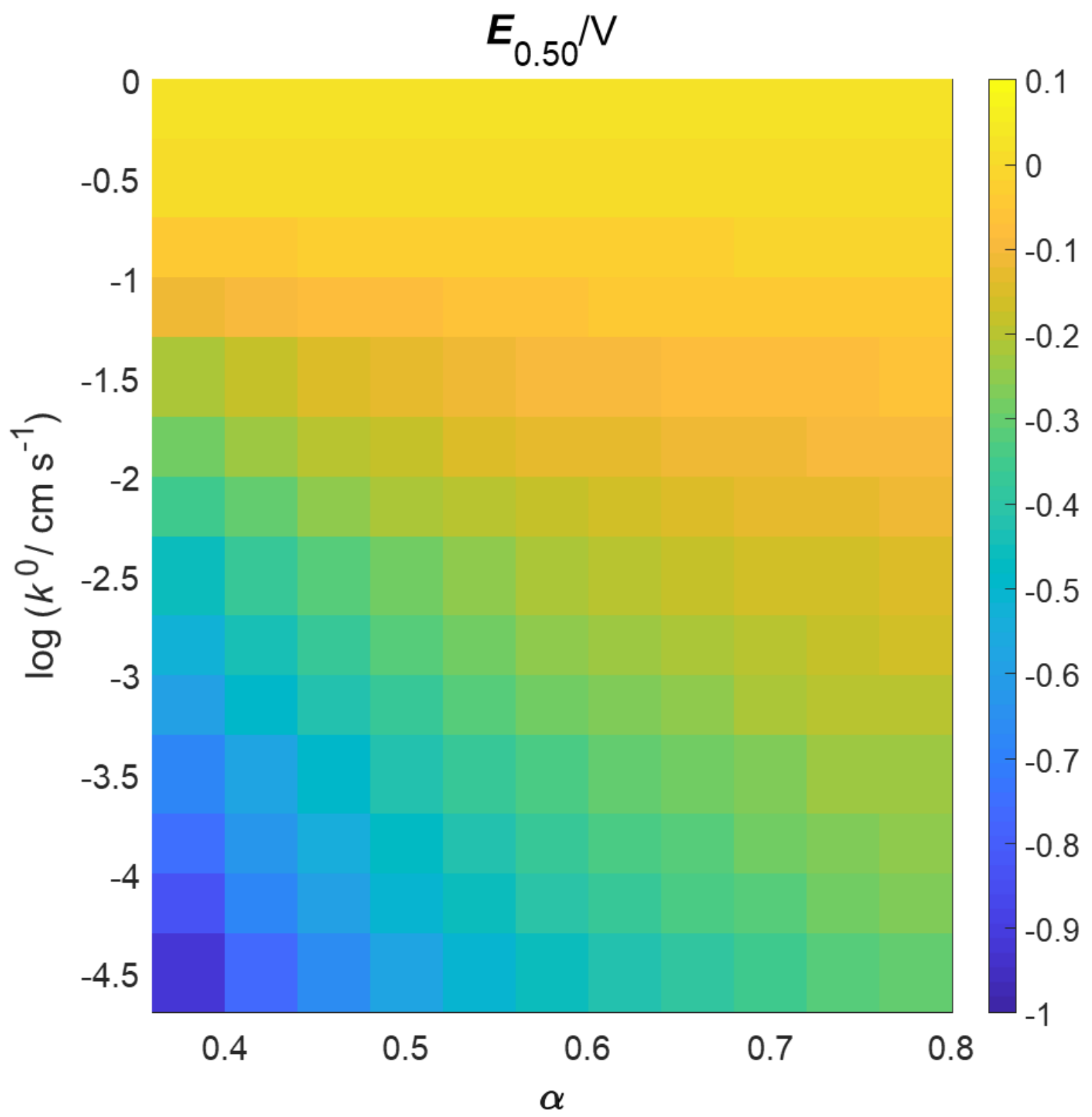

Figure S11. Effect of $\alpha$ and $k^{0}$ on the potential corresponding to $50 \%$ of the limiting current $\left(E_{0.50}\right)$ from the simulated SECCM voltammograms. 


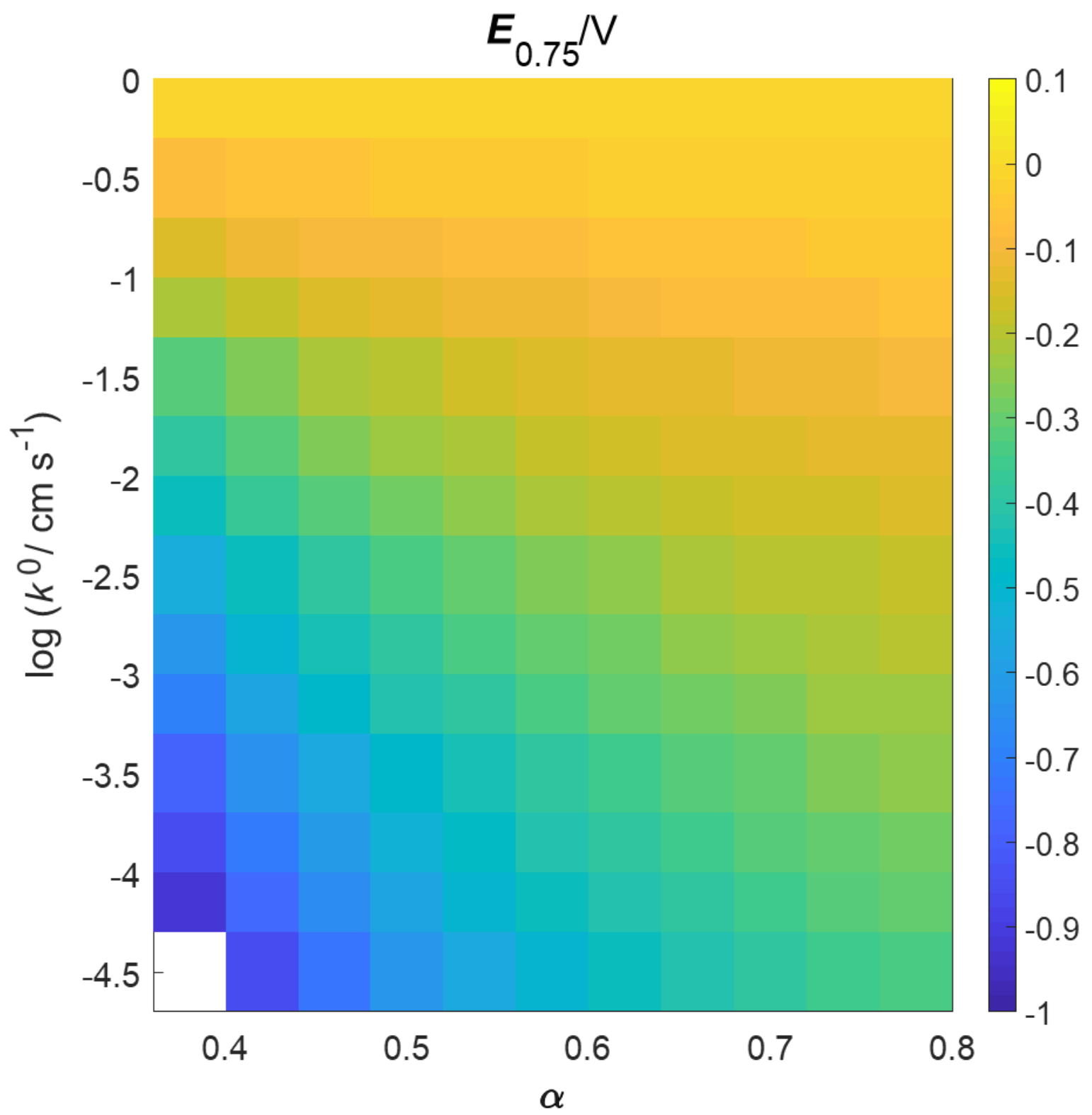

Figure S12. Effect of $\alpha$ and $k^{0}$ on the potential corresponding to $75 \%$ of the limiting current $\left(E_{0.75}\right)$ from the simulated SECCM voltammogram. The missing pixels at the bottom left corner result from $75 \%$ of the limiting current outside the simulated potential range $(0.5$ to $-1 \mathrm{~V})$. 


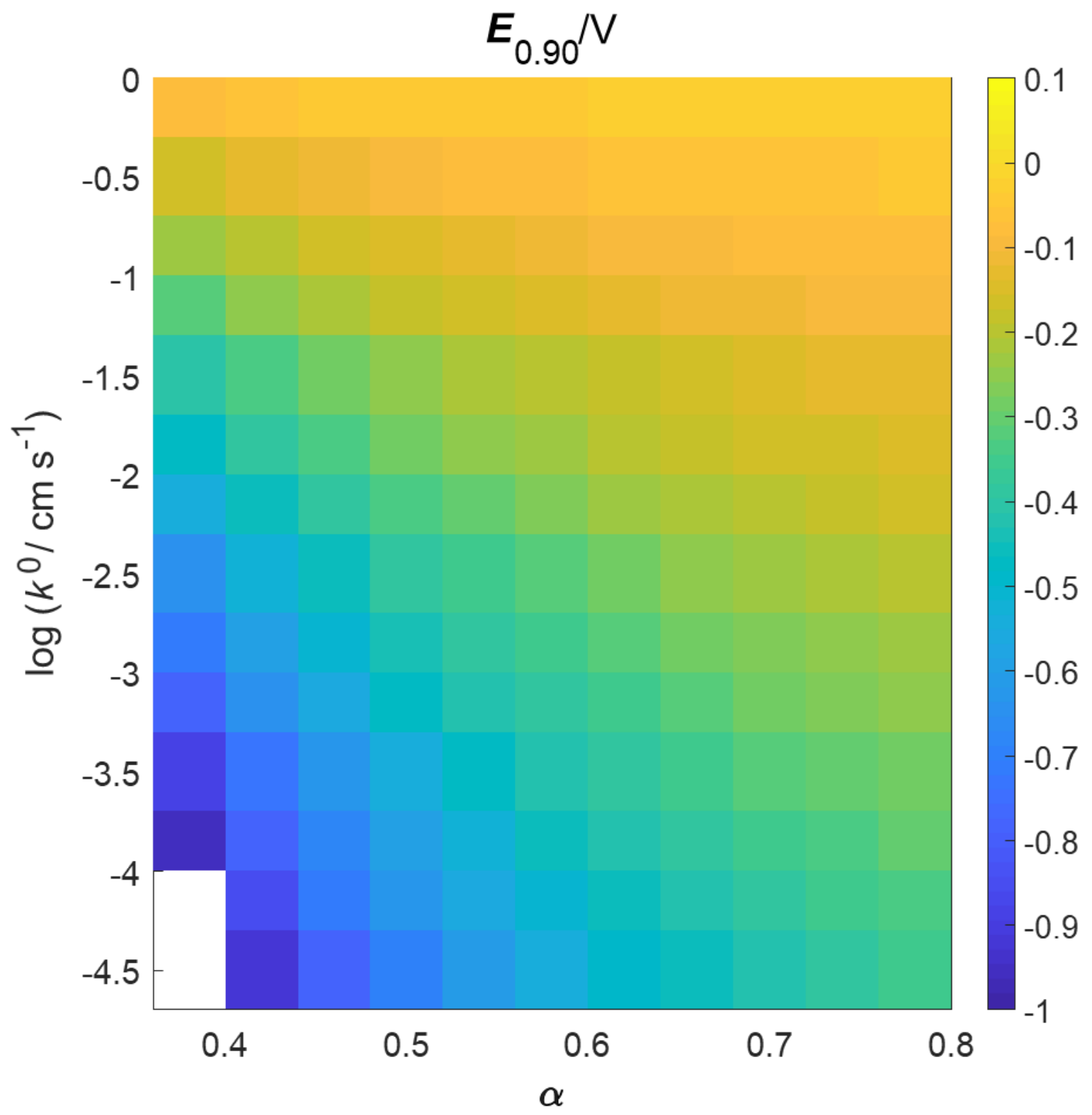

Figure S13. Effect of $\alpha$ and $k^{0}$ on the potential corresponding to $90 \%$ of the limiting current $\left(E_{0.90}\right)$ from the simulated SECCM voltammogram. The missing pixels at the bottom left corner result from $90 \%$ of the limiting current outside the simulated potential range $(0.5$ to $-1 \mathrm{~V})$. 

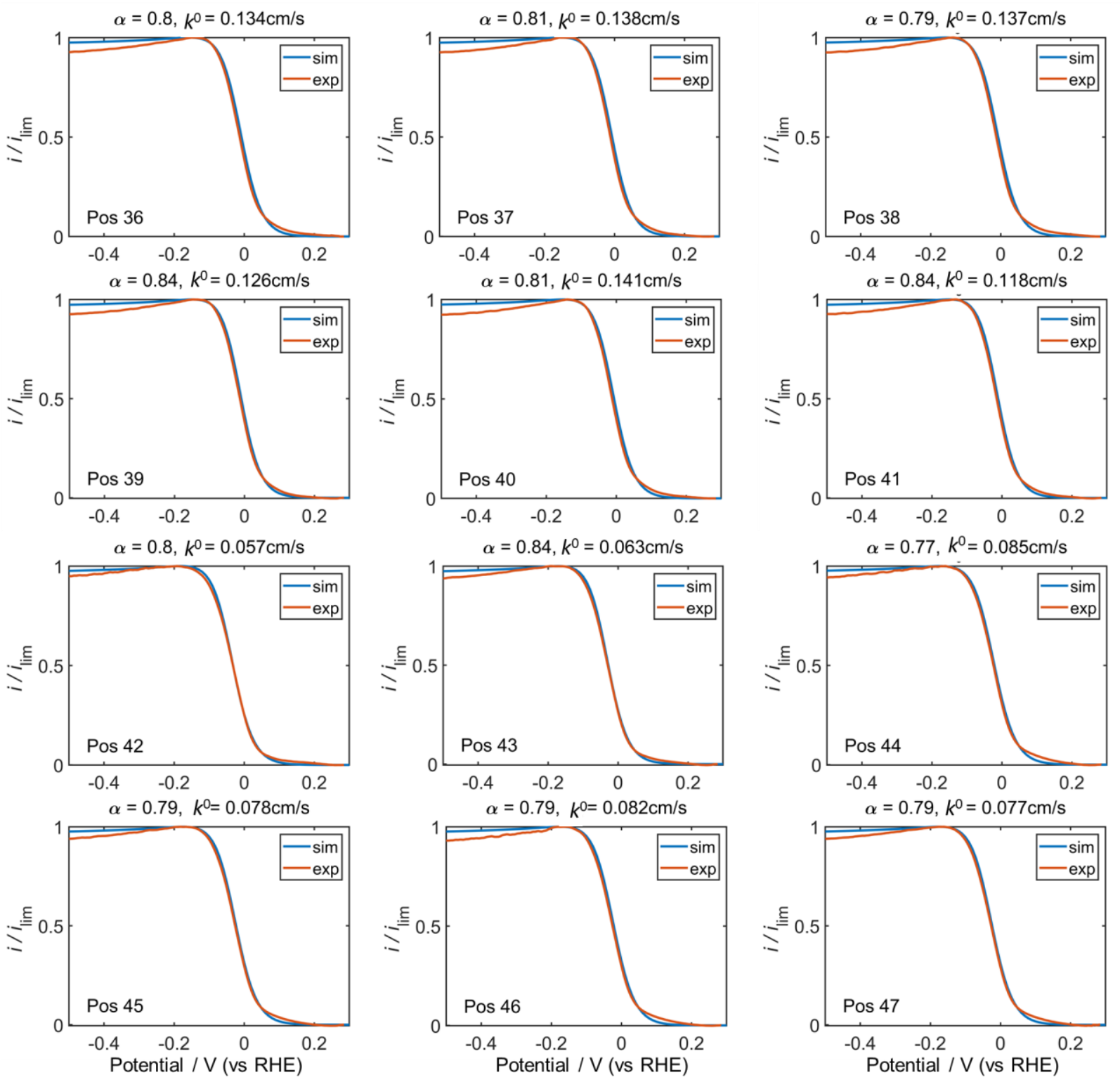

Figure S14. Experimental (orange) and simulated (blue) voltammograms (normalized) at 12 different places in an SECCM experiment of HER on polycrystalline Pt. The fitted kinetics parameters, $\alpha$ and $k^{0}$, were used to construct the maps shown in Figure S15. 
8. Additional correlated PZC and electroactivity maps on polycrystalline Pt

a
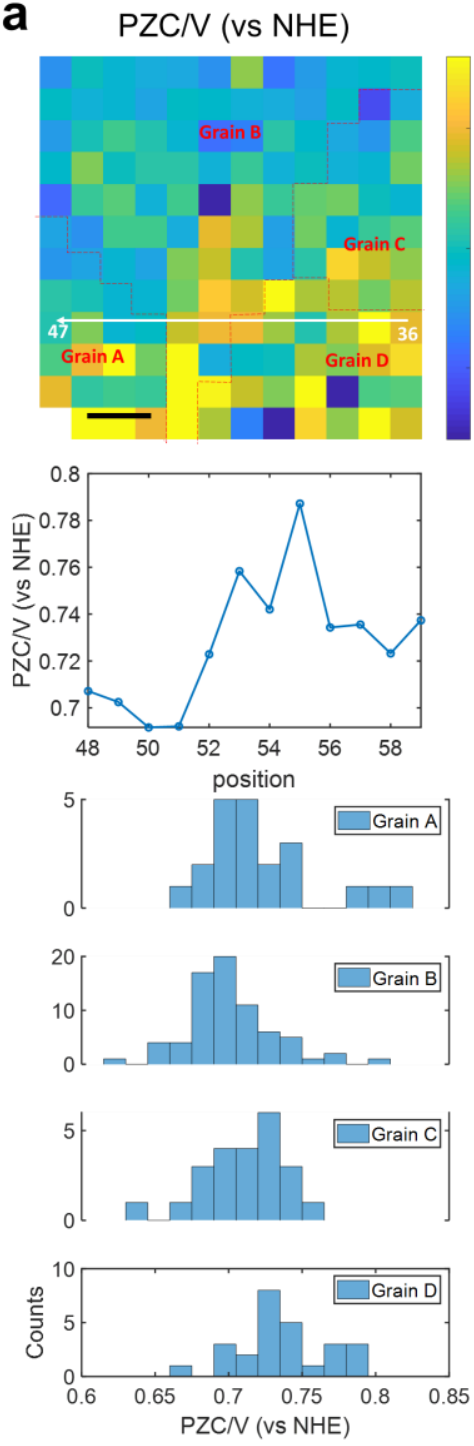

b

$k^{0} / \mathrm{cm} \mathrm{s}^{-1}$
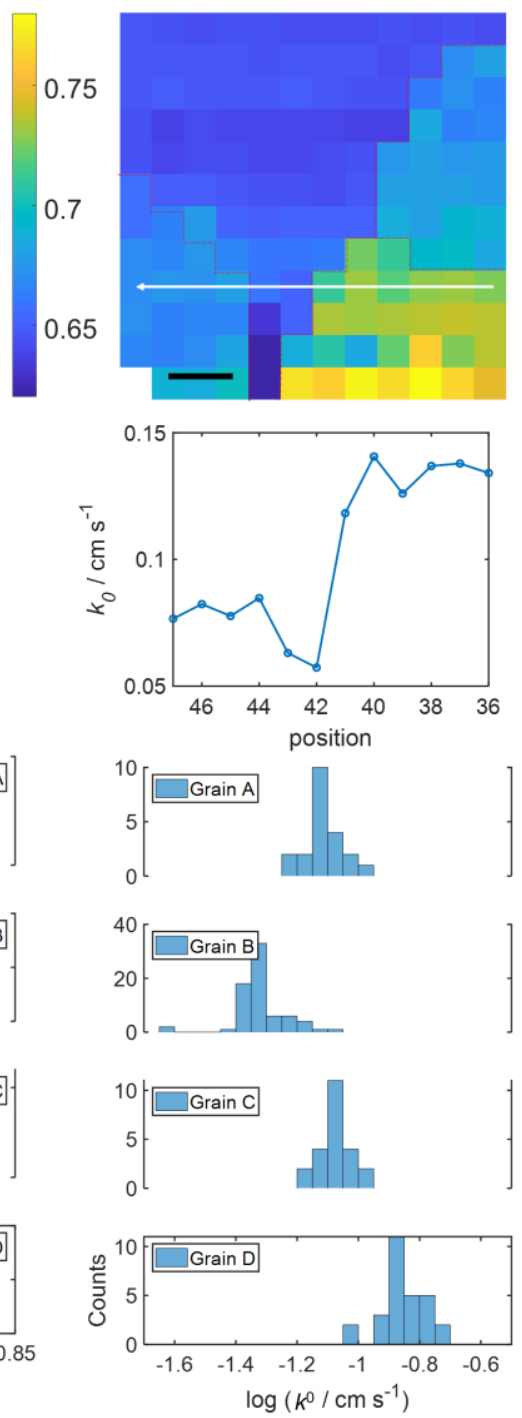

C

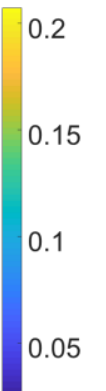

$\alpha$
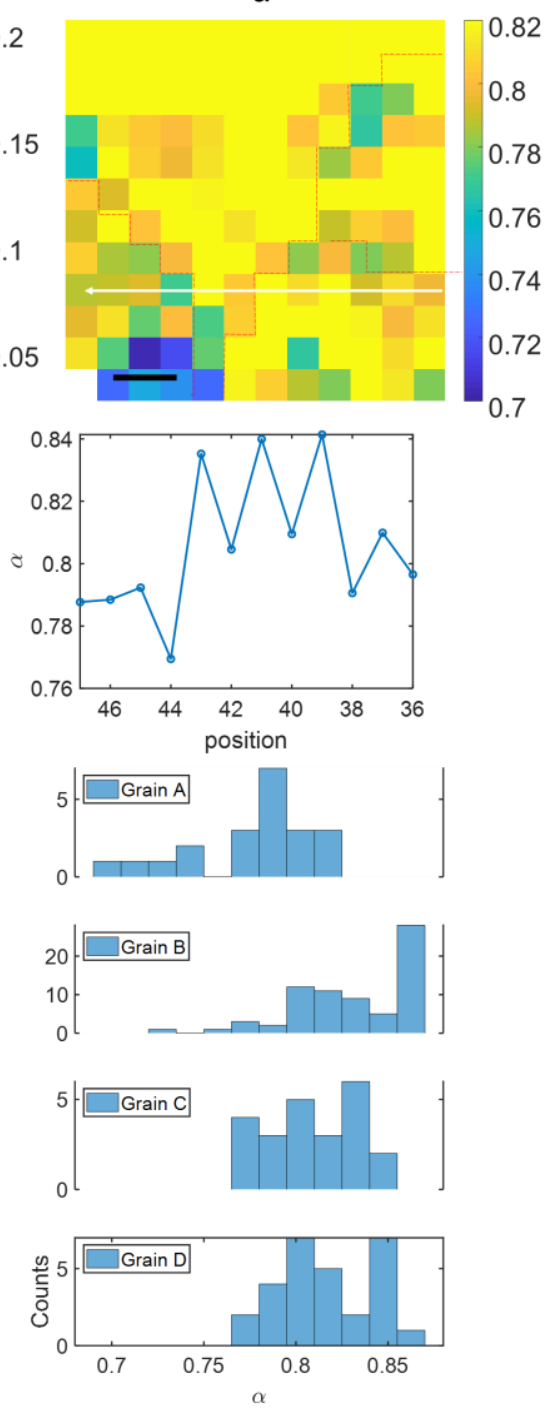

Figure S15. Heat map (top), line scan on the heat map (middle) and histograms based on the grains (bottom) for a) measured PZC, b) $k^{0}$ and c) $\alpha$ of HER at polycrystalline Pt in $10 \mathrm{mM} \mathrm{HClO}_{4}$ and $10 \mathrm{mM} \mathrm{KCl} . k^{0}$ and $\alpha$ were obtained by fitting the experimental voltammograms to simulated voltammograms using finite element simulation as shown in Section 6. The voltammograms from experiment and simulation corresponding to the line scan (white arrow in the maps) are plotted in Figure S14. The scale bars are is 10 $\mu \mathrm{m}$. The corresponding voltammetric movie is shown as Movie S2. 


\section{Captions for voltammetric movies}

Movie S1 Voltammetric movies of HER on polycrystalline Pt in $10 \mathrm{mM} \mathrm{HClO}_{4}$ and 10 $\mathrm{mM} \mathrm{KCl}$. The corresponding kinetic and PZC maps are shown in Figure 4.

Movie S2 Voltammetric movies of HER on polycrystalline Pt in $10 \mathrm{mM} \mathrm{HClO}_{4}$ and 10 $\mathrm{mM} \mathrm{KCl}$. The corresponding kinetic and PZC maps are shown in Figure S15. 


\section{References}

1. Pajkossy, T.; Kolb, D. M., Double layer capacitance of Pt(111) single crystal electrodes. Electrochim. Acta 2001, 46 (20), 3063-3071.

2. Hamelin, A., The surface state and the potential of zero charge of gold (100): a further assessment. J. Electroanal. Chem. 1995, 386 (1), 1-10. 